\title{
„Chemobrain“? Beeinträchtigungen kognitiver Funktionen bei Mammakarzinompatientinnen
}

Viele Patientinnen mit einem Mammakarzinom klagen über Beeinträchtigungen des Gedächtnisses, der Aufmerksamkeit und anderer kognitiver Funktionen. Lange Zeit bestand weitgehend Konsens darüber, dass solche Probleme durch die Chemotherapie verursacht werden. Laborexperimente und Tiermodelle fanden Mechanismen, durch die Zytostatika das Gehirn schädigen könnten. Neuere klinische Studien allerdings lassen daran zweifeln, dass das sogenannte Chemobrain überhaupt existiert.

Ein Buch zu lesen oder einem Gespräch zu folgen macht Mühe, das richtige Wort oder ein Name lassen sich nicht aus dem Gedächtnis abrufen, die Organisation des Alltags ist eine Überforderung und alle intellektuellen Leistungen sind ungewohnt anstrengend - solche Klagen hören viele Ärzte von ihren onkologischen Patienten. Die Beeinträchtigungen kognitiver Funktionen erreichen zwar bei Weitem nicht das Ausmaß einer Demenz, sondern bleiben im Bereich von „slips and lapses“, die auch jedem Gesunden passieren können [1], aber einige der Betroffenen fühlen sich erheblich beeinträchtigt [2].

\section{Evidenz für das}

\section{"Chemobrain"}

Die Ursache der Beschwerden schien gefunden, als im Jahr 1995 eine Studie bei $75 \%$ von 28 neuropsychologisch getesteten Brustkrebspatientinnen, die alle eine Chemotherapie hinter sich hatten, mäßige kognitive Beeinträchtigung feststellte [3] und eine Reihe weiterer Untersuchungen diesen Befund weitgehend bestätigte. Die Resultate der nachfolgenden Studien waren zwar nicht ganz so gravierend, doch auch hier zeigten Subgruppen von Patientinnen, die wegen eines nicht metastasierten Mammakarzinoms mit Chemotherapie behandelt worden waren, auffällig schlechte Leistungen in neuropsychologi- schen Tests. Meist war ungefähr ein Drittel der Patientinnen betroffen. Allerdings ergab sich kein einheitliches Störungsbild, sondern je nach Studie erschienen unterschiedliche kognitive Domänen beeinträchtigt. Zudem waren methodische Schwächen der Studien offensichtlich [4]. Befunde aus Studien mit Bildgebung des Gehirns, die meisten davon ebenfalls mit Mammakarzinompatientinnen, ergänzten die Ergebnisse der neuropsychologischen Studien: Nach einer Chemotherapie wurden abweichende Aktivierungsmuster und strukturelle Auffälligkeiten wie kleinere regionale Gehirnvolumina und eine geringere Integrität der weißen Substanz festgestellt. Auch die Ergebnisse dieser Studien waren sehr heterogen [5].

Trotz ihrer Unterschiedlichkeit im Detail schienen alle Befunde gleichermaßen auf die Chemotherapie als Ursache der von Patientinnen berichteten kognitiven Beeinträchtigungen hinzuweisen. Dazu kamen Studien im Tiermodell, die in ihrer Mehrzahl ebenfalls darauf schließen ließen, dass Zytostatika das Gehirn und kognitive Funktionen schädigen können [6]. Laborexperimente legten plausible Mechanismen der Schädigung nahe [7], und so setzte sich Mitte der Nullerjahre die Überzeugung durch, dass Chemotherapie bei einer großen Subgruppe der Mammakarzinompatientinnen - und anderer, weniger gut untersuchter Patienten mit Malignomen ohne ZNS-Beteiligung - zu erheblichen Beeinträchtigungen kognitiver Funktionen führt [4]. Das „Chemobrain“ gewann vor allem in den USA große Popularität in den Medien und der Öffentlichkeit.

\section{Widersprechende Befunde neuerer Studien}

Erst als sich das Konzept des Chemobrain schon fest etabliert hatte, erschienen vor 15 Jahren neben Querschnittstudien endlich auch prospektive Längsschnittstudien zum Thema. Allgemein war erwartet wor- den, dass die Längsschnittstudien bestehende Überzeugungen bestätigen und methodisch absichern würden - aber das Gegenteil passierte: Eine lange Reihe prospektiver Studien fand bereits vor Beginn einer Chemotherapie Auffälligkeiten kognitiver Funktionen [8-11]. Offensichtlich war zumindest ein Teil der kognitiven Beeinträchtigungen, die in Querschnittstudien nach einer Chemotherapie festgestellt worden waren, fälschlich als Folge der Chemotherapie aufgefasst worden tatsächlich hatten sie bereits vor Beginn der systemischen Therapie bestanden. Noch dazu zeigten auch Patientinnen, die ohne Chemotherapie behandelt wurden, beeinträchtigte Leistungen in neuropsychologischen Tests, und Studien mit Bildgebung fanden bereits vor Beginn einer Chemotherapie Auffälligkeiten des Gehirns [12-15]. Aus diesen Befunden geht hervor, dass Chemotherapie nicht die singuläre Ursache kognitiver Beeinträchtigungen bei Krebspatienten sein kann wenn Chemotherapie überhaupt eine Rolle spielt, dann neben anderen Faktoren, von denen zumindest ein Teil schon vor Behandlungsbeginn wirkt.

Die neuesten Metaanalysen stimmen darin überein, dass Störungen kognitiver Funktionen bei Mammakarzinompatientinnen mild sind und unabhängig von einer Chemotherapie auftreten $[16,17]$. Trotzdem ist die Annahme, Chemotherapie sei die wesentliche Ursache der von Mammakarzinompatientinnen erlebten kognitiven Beeinträchtigungen, sowohl unter Wissenschaftlern als auch in den Medien weiterhin sehr verbreitet.

\section{Andere Faktoren in der Verursachung kognitiver Beeinträchtigungen}

Neben der Chemotherapie geriet die endokrine Therapie des Mammakarzinoms in den Verdacht, Störungen kognitiver 


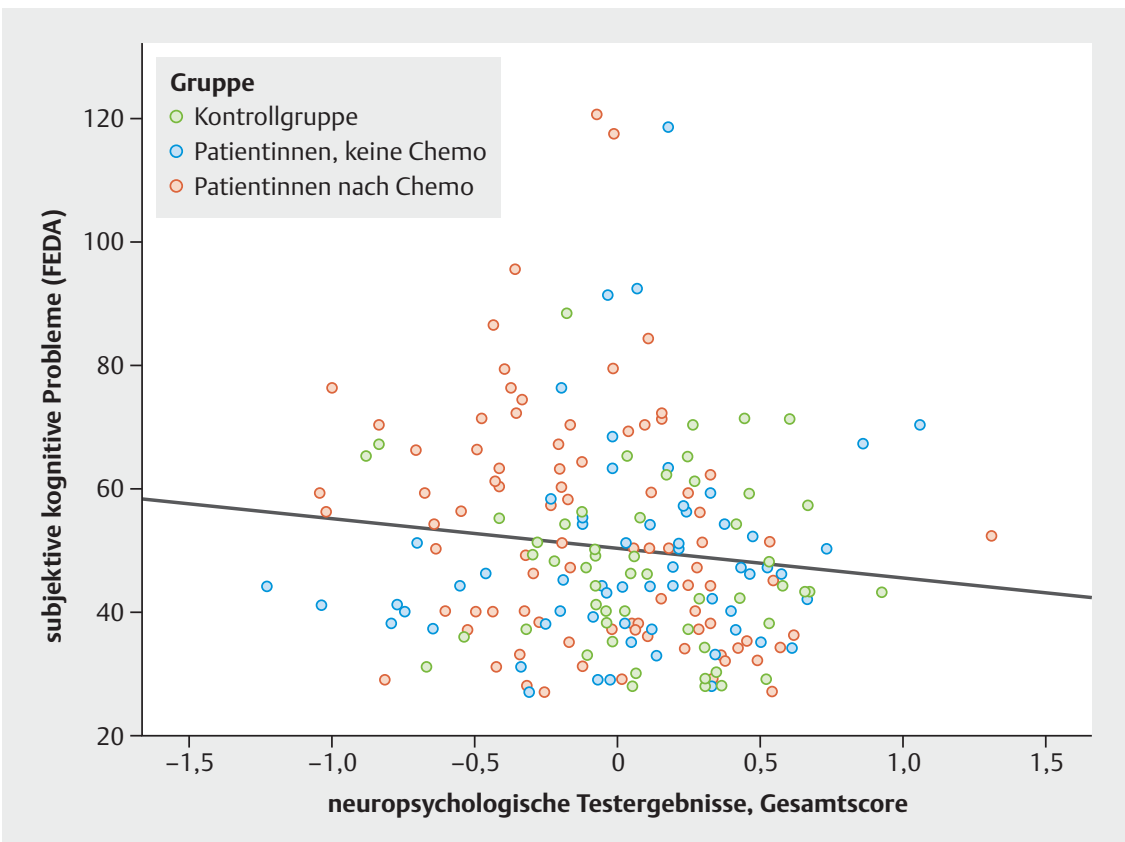

- Abb. 1 Subjektive kognitive Probleme und kognitive Testleistungen (Gesamtscore) 1 Jahr nach der Diagnose bei Mammakarzinompatientinnen mit und ohne Chemotherapie und einer Kontrollgruppe nicht an Krebs erkrankter Frauen. FEDA: Fragebogen erlebter Defizite der Aufmerksamkeit, höhere Werte stehen für größere subjektive kognitive Probleme. Der Gesamtscore wurde für Alter und Bildung korrigiert und z-standardisiert, höhere Werte stehen für bessere Leistungen. Der Zusammenhang ist statistisch signifikant (Kendall's $\mathrm{T}=-0,11$; $\mathrm{p}=0,02 ; \mathrm{n}=206$ ). Die Grafik zeigt Daten der Studie Cognicares [27].

Funktionen zu verursachen. Viele Studien haben Effekte von Tamoxifen und Aromatase-Inhibitoren auf kognitive Funktionen verglichen - mit unterschiedlichen Ergebnissen. Nur sehr wenige Studien haben die Auswirkungen einer therapieinduzierten vorzeitigen Menopause untersucht und fanden kaum [18] oder gar keine [19, 20] Konsequenzen für kognitive Funktionen. In einer aktuellen prospektiven Studie wurden während einer Nachbeobachtungszeit von bis zu 6 Jahren keinerlei negative Effekte endokriner Therapie auf kognitive Funktionen festgestellt [21]. Zu einem ähnlichen Schluss kommt eine neue Metaanalyse: Zwar fanden sich - wenige querschnittliche Unterschiede zwischen Patientinnen mit und ohne endokrine Behandlung; wurden aber in Längsschnittstudien Veränderungen kognitiver Funktionen betrachtet, zeigten sich keine Effekte endokriner Therapie [22].

Zu kognitiven Störungen vor Therapiebeginn werden mehrere Hypothesen diskutiert:
- Krebs und kognitive Störungen könnten auf eine gemeinsame genetische Vulnerabilität zurückgehen. Es gibt keine empirische Evidenz und kaum eine theoretische Begründung für diese Hypothese. Zurzeit sieht es im Gegenteil eher so aus, als hätten Überlebende einer Krebserkrankung ein besonders niedriges Risiko, an einer Demenz zu erkranken [23].

- Die Krebserkrankung selbst könnte zu Beeinträchtigungen des Gehirns führen, möglicherweise durch eine Entzündungsreaktion mit erhöhter Ausschüttung von Zytokinen. Diese Hypothese wurde bereits vielfach untersucht, aber eindeutige Ergebnisse liegen noch nicht vor [7].

- Schließlich könnte psychische Belastung durch die Erkrankung und die Therapie die kognitive Leistungsfähigkeit herabsetzen. Stress greift tief in die Arbeitsweise des Gehirns ein [24], und posttraumatischer Stress führt zu solchen funktionellen und strukturellen Veränderungen des Gehirns [25], wie sie bei Brustkrebspatientinnen beobachtet wurden. Eine aktuelle Übersichtsarbeit stellt fest, dass Stress bei Mammakarzinompatientinnen mit Beeinträchtigungen kognitiver Funktionen assoziiert ist [26]. Zwar haben viele Studien keinen Zusammenhang zwischen psychischer Belastung und kognitiven Funktionen gefunden - in diesen Studien wurden aber Angst, Depression und andere Indikatoren psychischer Belastung nur mit kurzen Selbstbericht-Fragebögen erfasst, deren Ergebnisse möglicherweise zu ungenau und durch subjektive Bewertungen der Patientinnen verzerrt sind. Wir haben in einer prospektiven Längsschnittstudie die Auswirkungen von erkrankungsbedingtem posttraumatischem Stress auf kognitive Funktionen bei Mammakarzinompatientinnen untersucht. Posttraumatische Symptomatik wurde nicht von den Patientinnen selbst eingeschätzt, sondern klinisch diagnostiziert. Unsere Patientinnen zeigten sowohl vor Therapiebeginn als auch ein Jahr später minimale kognitive Beeinträchtigungen, die unabhängig von einer Chemotherapie auftraten und sich großenteils durch posttraumatischen Stress erklären ließen [9, 27].

Neben der etablierten „pharmako-toxikologischen“ Sichtweise, die kognitive Störungen bei Krebspatienten auf Nebenwirkungen von Medikamenten zurückführt, setzt sich allmählich eine multifaktorielle Perspektive durch: Das Zusammenspiel einer Vielzahl von Faktoren - Einflüsse durch Erkrankung und Therapie, aber auch Alter, Bildung, Lebensstil, psychische Belastung und ihre vielen somatischen Folgen, Komorbiditäten, genetische Ausstattung - könnte die kognitive Leistungsfähigkeit bei Krebspatienten determinieren [23]. Es stellt sich darüber hinaus die Frage, ob kognitive Beeinträchtigungen im Zusammenhang mit Krebs tatsächlich ein spezifisches Problem sind. Möglicherweise wirkt sich eine Krebserkrankung auf kognitive Funktionen nicht anders aus als andere extrem belastende Ereignisse auch. 


\section{Subjektive kognitive \\ Probleme vs. objektiv mess- bare Beeinträchtigungen}

Während objektiv messbare kognitive Beeinträchtigungen im Zusammenhang mit einem Mammakarzinom mild sind, berichten Patientinnen manchmal von dramatischen kognitiven Problemen - objektive und subjektive kognitive Störungen passen nicht gut zueinander. Erstaunlicherweise werden tatsächlich regelmäßig keine oder nur schwache statistische Zusammenhänge zwischen objektiv messbaren und subjektiven, von den Patientinnen selbst berichteten kognitiven Störungen gefunden [28] ( $\triangleright$ Abb. 1). Mit anderen Worten: Die Patientinnen, die über kognitive Probleme klagen, sind nicht dieselben, die in neuropsychologischen Tests auffällige Ergebnisse zeigen; zwischen diesen beiden Gruppen gibt es nicht viel mehr als nur Zufallsüberschneidungen. Was für objektiv messbare kognitive Beeinträchtigungen diskutiert wird, ist für subjektive kognitive Probleme eindeutig nachgewiesen: Sie sind mit dem psychischen Befinden assoziiert [28] ( $\bullet$ Abb. 2). Der Zusammenhang zwischen subjektiven kognitiven Problemen mit Angst, Depressivität und anderen psychischen Problemen findet sich konsistent in nahezu allen Studien und ist eines der robustesten Ergebnisse der Forschung zu kognitiven Beeinträchtigungen im Zusammenhang mit einer Krebserkrankung.

Zur Diskrepanz zwischen objektiven und subjektiven kognitiven Störungen sind viele Erklärungsansätze entwickelt und getestet worden - ohne befriedigendes Ergebnis. Es herrscht Uneinigkeit darüber, ob diese Diskrepanz ein Methodenartefakt ist oder ob es sich bei subjektiven Klagen und objektiven Beeinträchtigen tatsächlich um zwei verschiedene Phänomene handelt. Möglicherweise spielen subjektive Bewertungsstile der Patientinnen - wie im Extremfall Katastrophisieren oder Minimisieren - eine Rolle: Sie wirken sich sowohl auf selbstberichtete kognitive Probleme als auch auf selbstberichtete Depressivität und Angst etc. aus, aber nicht auf objektive Testergebnisse. So könnten Bewertungsstile der Patientinnen den Zusammenhang von subjektiv berichteten kogni-

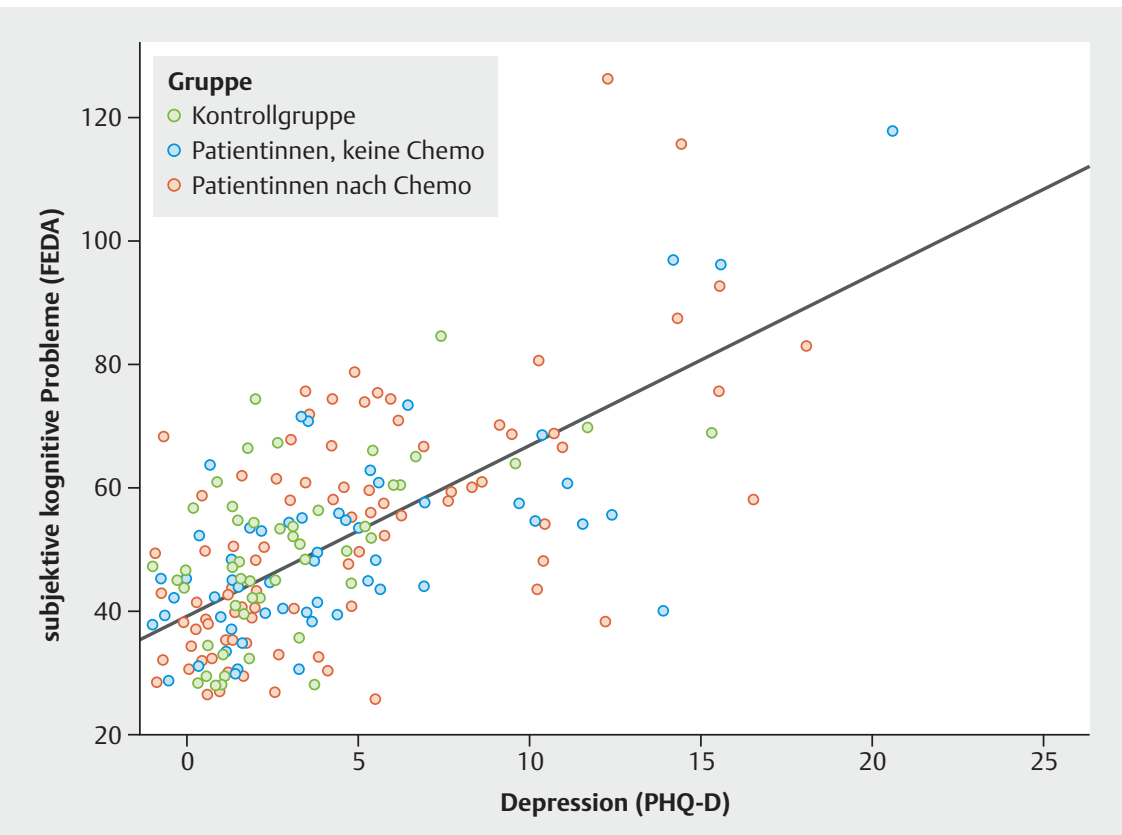

- Abb. 2 Subjektive kognitive Probleme und Depression 1 Jahr nach der Diagnose bei Mammakarzinompatientinnen mit und ohne Chemotherapie und einer Kontrollgruppe nicht an Krebs erkrankter Frauen. FEDA: Fragebogen erlebter Defizite der Aufmerksamkeit, höhere Werte stehen für größere subjektive kognitive Probleme. PHQ-D: Patient Health Questionnaire, deutsche Version, höhere Werte stehen für höhere Depressivität. Die Datenpunkte sind leicht verschoben dargestellt, um Überlagerungen zu vermeiden. Der Zusammenhang ist statistisch signifikant (Kendall's $\mathrm{T}=0,48 ; \mathrm{p}<0,001 ; \mathrm{n}=204$ ). Die Grafik zeigt Daten der Studie Cognicares [27].

tiven Problemen mit objektiv gemessenen kognitiven Beeinträchtigungen maskieren.

\section{Klagen über kognitive Probleme in der Praxis - Aufklärung und Inter- ventionen}

Bei der Aufklärung zu den Folgen von Erkrankung und Therapie für kognitive Funktionen ergibt sich ein Dilemma: Einerseits sollten Patientinnen über dieses relevante Problem informiert werden, andererseits kann die Information nachweislich zu Nocebo-Effekten führen [29]. Wenn Patientinnen zum sogenannten Chemobrain aufgeklärt werden, sollten sie unbedingt erfahren, dass das Problem genauso auch bei Patientinnen ohne Chemotherapie und sogar schon vor Behandlungsbeginn auftritt.

Wenn Patientinnen über kognitive Beeinträchtigungen klagen, sollten Ärzte be- denken, dass solche Beschwerden eng mit Depression, Angst und allgemein psychischer Belastung zusammenhängen [28]. Das ist sicher kein Grund, die Klagen nicht ernst zu nehmen - im Gegenteil; den Patientinnen sollte Hilfe angeboten werden. Zunächst ist es sicher sinnvoll, eine Psychoonkologin hinzuzuziehen, damit die kognitive Problematik nicht isoliert, sondern im Kontext der psychischen Situation der Patientin betrachtet werden kann.

Eine aktuelle Übersichtsarbeit hat festgestellt, dass eine ganze Reihe von Interventionen bei subjektiven kognitiven Beeinträchtigungen erfolgreich wirkt, darunter kognitives Training und kognitive Rehabilitation mit Vermittlung kompensatorischer Strategien, aber auch Mindfulnessbased Stress Reduction und körperliche Bewegung, insbesondere Yoga, Qigong und Tai-Chi [7]. Für keine dieser Interventionen ist allerdings bisher nachgewiesen, dass sie auch neuropsychologisch messbare kognitive Funktionen verbessert. Der Einsatz von Stimulanzien wie Methylpheni- 
dat und Modafinil, von Antidementiva wie Donepezil und von Antidepressiva wurde und wird getestet, ist aber zurzeit außerhalb von Studien nicht empfohlen [7].

\section{FAZIT}

Neuropsychologisch nachweisbare Beeinträchtigungen kognitiver Funktionen bei Patientinnen mit nicht metastasiertem Mammakarzinom sind mild. Sie können schon vor Behandlungsbeginn und unabhängig von einer Chemotherapie auftreten. Wahrscheinlich sind sie multifaktoriell verursacht. Subjektive kognitive Probleme sind verbreitet und manchmal dramatisch. Sie hängen mit Depressivität, Angst und psychischer Belastung, aber wenig oder gar nicht mit objektiv messbaren Beeinträchtigungen zusammen.

\section{Interessenkonflikt}

Die Autoren geben an, dass kein Interessenkonflikt besteht.

\section{Autorinnen/Autor}

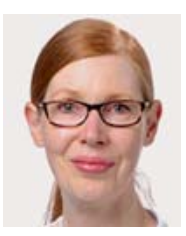

\section{Kerstin Hermelink}

PD Dr. rer. biol. hum. habil., Leitende Psychologin in der Klinik und Poliklinik für Frauenheilkunde und Geburtshilfe am Universitätsklinikum München

\section{Korrespondenzadresse}

PD Dr. rer. biol. hum. habil. Kerstin Hermelink

Klinikum der Universität München Klinik und Poliklinik für Frauenheilkunde und Geburtshilfe

Marchioninistraße 15 81377 München

Kerstin.Hermelink@med.uni-muenchen.de

\section{Literatur}

[1] Shilling V, Jenkins V. Self-reported cognitive problems in women receiving adjuvant therapy for breast cancer. Eur J Oncol Nurs 2007; 11: 6-15

[2] Von Ah D, Habermann B, Carpenter JS et al. Impact of perceived cognitive impairment in breast cancer survivors. Eur J Oncol Nurs 2013; 17: 236-241. doi:10.1016/j. ejon.2012.06.002

[3] Wieneke MH, Dienst ER. Neuropsychological assessment of cognitive functioning following chemotherapy for breast cancer. Psycho-Oncology 1995; 4: 61-66

[4] Shilling V, Jenkins V, Trapala IS. The (mis)classification of chemo-fog - methodological inconsistencies in the investigation of cognitive impairment after chemotherapy. Breast Cancer Res Treat 2006; 95: 125-129

[5] Pomykala KL, de Ruiter MB, Deprez S et al. Integrating imaging findings in evaluating the post-chemotherapy brain. Brain Imaging Behav 2013; 7: 436-452. doi:10.1007/s11682013-9239-y

[6] Seigers R, Schagen SB, Van Tellingen O et al. Chemotherapy-related cognitive dysfunction: current animal studies and future directions. Brain Imaging Behav 2013; 7: 453459. doi:10.1007/s11682-013-9250-3

[7] Chung NC, Walker AK, Dhillon HM et al. Mechanisms and Treatment for Cancer- and Chemotherapy-Related Cognitive Impairment in Survivors of Non-CNS Malignancies. Oncology (Williston Park) 2018; 32: $591-$ 598

[8] Ahles TA, Saykin AJ, McDonald BC et al. Cognitive function in breast cancer patients prior to adjuvant treatment. Breast Cancer Res Treat 2008; 110: 143-152

[9] Hermelink K, Voigt V, Kaste J et al. Elucidating pretreatment cognitive impairment in breast cancer patients: the impact of cancer-related post-traumatic stress. J Natl Cancer Inst 2015; 107: djv099. doi:10.1093/jnci/ djv099

[10] Wefel JS, Lenzi R, Theriault R et al. 'Chemobrain' in breast carcinoma?: a prologue. Cancer 2004; 101: 466-475

[11] Hermelink K, Untch M, Lux MP et al. Cognitive function during neoadjuvant chemotherapy for breast cancer: results of a prospective, multicenter, longitudinal study. Cancer 2007; 109: 1905-1913

[12] Cimprich B, Reuter-Lorenz P, Nelson J et al. Prechemotherapy alterations in brain function in women with breast cancer. J Clin Exp Neuropsychol 2010; 32: 324-331. doi:10.1080/13803390903032537
[13] Scherling C, Collins B, Mackenzie J et al. Prechemotherapy differences in response inhibition in breast cancer patients compared to controls: a functional magnetic resonance imaging study. J Clin Exp Neuropsychol 2012; 34: 543-560. doi:10.1080/ 13803395.2012 .666227

[14] Lopez Zunini RA, Scherling C, Wallis N et al. Differences in verbal memory retrieval in breast cancer chemotherapy patients compared to healthy controls: a prospective fMRI study. Brain Imaging Behav 2013; 7: 460477. doi:10.1007/s11682-012-9213-0

[15] McDonald BC, Conroy SK, Ahles TA et al. Alterations in brain activation during working memory processing associated with breast cancer and treatment: a prospective functional magnetic resonance imaging study. J Clin Oncol 2012; 30: 2500-2508. doi:10.1200/JCO.2011.38.5674

[16] Bernstein LJ, McCreath GA, Komeylian Z et al. Cognitive impairment in breast cancer survivors treated with chemotherapy depends on control group type and cognitive domains assessed: A multilevel meta-analysis. Neurosci Biobehav Rev 2017; 83: 417-428. doi:10.1016/j.neubiorev.2017.10.028

[17] Ono M, Ogilvie JM, Wilson JS et al. A metaanalysis of cognitive impairment and decline associated with adjuvant chemotherapy in women with breast cancer. Front Oncol 2015; 5: 59. doi:10.3389/fonc.2015.00059

[18] Vearncombe KJ, Rolfe M, Andrew B et al. Cognitive effects of chemotherapy-induced menopause in breast cancer. Clin Neuropsychol 2011; 25: 1295-1313. doi:10.1080/ 13854046.2011.631586

[19] Hermelink K, Henschel V, Untch $M$ et al. Short-term effects of treatment-induced hormonal changes on cognitive function in breast cancer patients: results of a multicenter, prospective, longitudinal study. Cancer 2008; 113: 2431-2439

[20] Phillips KA, Regan MM, Ribi K et al. Adjuvant ovarian function suppression and cognitive function in women with breast cancer. $\mathrm{Br}$ J Cancer 2016; 114: 956-964. doi:10.1038/ bjc. 2016.71

[21] Van Dyk K, Crespi CM, Bower JE et al. The cognitive effects of endocrine therapy in survivors of breast cancer: A prospective longitudinal study up to 6 years after treatment. Cancer 2019; 125: 681-689. doi:10.1002/ cncr.31858

[22] Underwood EA, Rochon PA, Moineddin R et al. Cognitive sequelae of endocrine therapy in women treated for breast cancer: a metaanalysis. Breast Cancer Res Treat 2018; 168 : 299-310. doi:10.1007/s10549-017-4627-4 
[23] Ahles TA, Hurria A. New Challenges in Psycho-Oncology Research IV: Cognition and cancer: Conceptual and methodological issues and future directions. Psycho-Oncology 2018; 27: 3-9. doi:10.1002/pon.4564

[24] Andreotti C, Root JC, Ahles TA et al. Cancer. coping, and cognition: a model for the role of stress reactivity in cancer-related cognitive decline. Psycho-Oncology 2015; 24: 617-623. doi:10.1002/pon.3683

[25] Bremner JD. Traumatic stress: effects on the brain. Dialogues Clin Neurosci 2006; 8: 445461
[26] Yang Y, Hendrix CC. Cancer-Related Cognitive Impairment in Breast Cancer Patients: Influences of Psychological Variables. Asia Pac J Oncol Nurs 2018; 5: 296-306. doi:10.4103/apjon.apjon_16_18

[27] Hermelink K, Buhner M, Sckopke P et al. Chemotherapy and Post-traumatic Stress in the Causation of Cognitive Dysfunction in Breast Cancer Patients. J Natl Cancer Inst 2017; 109: djx057. doi:10.1093/jnci/djx057

[28] Bray VJ, Dhillon HM, Vardy JL. Systematic review of self-reported cognitive function in cancer patients following chemotherapy treatment. J Cancer Surviv 2018; 12: 537559. doi:10.1007/s11764-018-0692-x
[29] Schagen SB, Das E, Vermeulen I. Information about chemotherapy-associated cognitive problems contributes to cognitive problems in cancer patients. Psycho-Oncology 2012; 21: 1132-1135. doi:10.1002/pon.2011

\section{Bibliografie}

DOI https://doi.org/10.1055/a-0825-8384

Geburtsh Frauenheilk 2019; 79: 461-465 @ Georg Thieme Verlag KG Stuttgart · New York । ISSN 0016-5751 